\title{
Insertion of Degradable Thioester Linkages into Styrene and Methacrylate Polymers
}

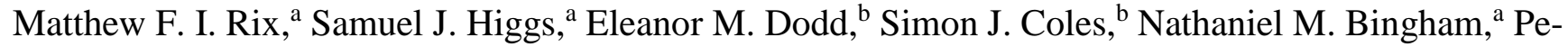 \\ ter J. Roth ${ }^{\mathrm{a}, *}$ \\ a Department of Chemistry, School of Chemistry and Chemical Engineering, University of Surrey, Guildford, GU2 7XH, \\ UK; ${ }^{b}$ National Crystallography Service, School of Chemistry, University of Southampton, Southampton, SO17 1BJ, UK
}

\section{Supporting Information Placeholder}

\begin{abstract}
The thionolactone 3,3-dimethyl-2,3-dihydro-5Hbenzo[e][1,4]dioxepine-5-thione (DBT) is shown to homopolymerize and, for the first time, copolymerize with styrene and methacrylates, introducing degradable thioester backbone functionality. The rapid copolymerization with styrene was exploited to produce copolymers through free-radical polymerization in a starve-fed semi-batch setup. The copolymerization of DBT with tert-butyl methacrylate under RAFT conditions was hypothesized to involve selective retardation of DBT-terminal chains that led to a more uniform distribution of degradable thioester linkages between chains. Surprisingly, the aminolysis of DBT homopolymers was accompanied by the intramolecular ether cleavage within the primary degradation product leading to the formation of 2,2dimethylthiirane and salicylamides.
\end{abstract}

The incorporation of backbone degradability into vinyl copolymers is a remaining frontier in the polymer chemistry arena and offers new prospects in biomedicine.1, 2 The radical ring-opening polymerization (RROP) of cyclic ketene acetals ${ }^{3}$ and allyl sulfide lactones ${ }^{4}$ has been used for several decades to install ester linkages into the backbone of vinyl copolymers. But these ester groups typically require harsh, non-selective conditions for cleavage. Recently, thionolactones were introduced as a new class of monomers undergoing radical ringopening polymerization. The proposed mechanism, termed thiocarbonyl addition-ring-opening (TARO), 5 involves the reversible 6 addition of a radical onto the thiocarbonyl group to give an intermediate $\left(-\mathrm{C}^{\prime}(\mathrm{SR}) \mathrm{O}^{-}\right)$radical, which undergoes irreversible ring-opening through $\beta$-scission to yield thioester $(-\mathrm{C}(=0) \mathrm{SR}-)$ backbone functionality. Significantly, these weak linkages can selectively be cleaved through aminolysis, thiolysis, or oxidative cleavage ${ }^{7}$ and are promising for the intracellular delivery of drugs through glutathione-triggered degradation. To date, three monomers, $\mathbf{1}$ and $\mathbf{2 a - b}$, have been shown to undergo TARO polymerization.
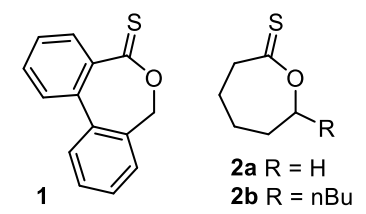

Dibenzo[c,e]oxepine(7H)-5-thione (DOT, 1), is incorporated rapidly into copolymerizations with secondary more activated monomers (acrylates, acrylamides, acrylonitrile, $N$-functional maleimides), while its homopolymerization is slow and lowyielding.5, 8, 9 Conversely, the $\varepsilon$-thionocaprolactones $2 \mathbf{2 a - b}$ undergo polymerization with the less activated monomers vinyl acetate and vinyl pivalate. ${ }^{10,11}$ Notably, TARO copolymerization with styrenic and methacrylic monomers has not been achieved.

Herein, a new monomer, 3,3-dimethyl-2,3-dihydro-5 $\mathrm{H}$ benzo[e][1,4]dioxepine-5-thione (DBT, 3) is presented that homopolymerized and copolymerized with styrene and methacrylates. DBT was synthesised in three steps from salicylic acid through phenol alkylation, lactonization, and thionation (see Scheme 1A and Figure S1-S9). DBT was hypothesised to be superior to DOT for two reasons. Firstly, the re-initiating radical (6) was chosen to resemble the tert-butyl radical which has initiation rate constants two orders of magnitude larger than the benzylic radical in DOT.12, 13 Secondly, the observed lack of copolymerization of DOT with styrene and methacrylates was attributed to the addition equilibrium lying on the side of the growing styrenic/methacrylic radical. In DBT, the ortho-alkoxy substituent was hypothesised to stabilise the intermediate radical through lone pair donation (resonance structure $\mathbf{5 b}$ in Scheme 1B). ${ }^{14}$ Indeed, preliminary ab initio molecular orbital theory and DFT calculations performed at the G3(MP2)-RAD(+) level estimated that the addition of a methyl radical onto DBT was $6.9 \mathrm{~kJ} / \mathrm{mol}$ more favorable than the addition of a methyl radical onto DOT, (see Figure S10. Notably, both changes (lowering the energy of the intermediate and increasing the energy of the open-ring radical) disfavor ring-opening. It was, however, hypothesized that the energy change associated with the thionolactone-thioester isomerization would be sufficient to drive the ring-opening. DBT was stable at room temperature for at least 25 days but when heated in refluxing xylene isomerized (51\% after $1.8 \mathrm{~h}$ ) to a 6-membered thionolactone, 4 , which was also identified as a minor impurity in crude DBT, see Figure S11-14. An attempted copolymerization of isolated $\mathbf{4}$ and methyl acrylate (MA) resulted in retardation without incorporation of thioesters. 
A-Synthesis of Thionolactone Monomer DBT

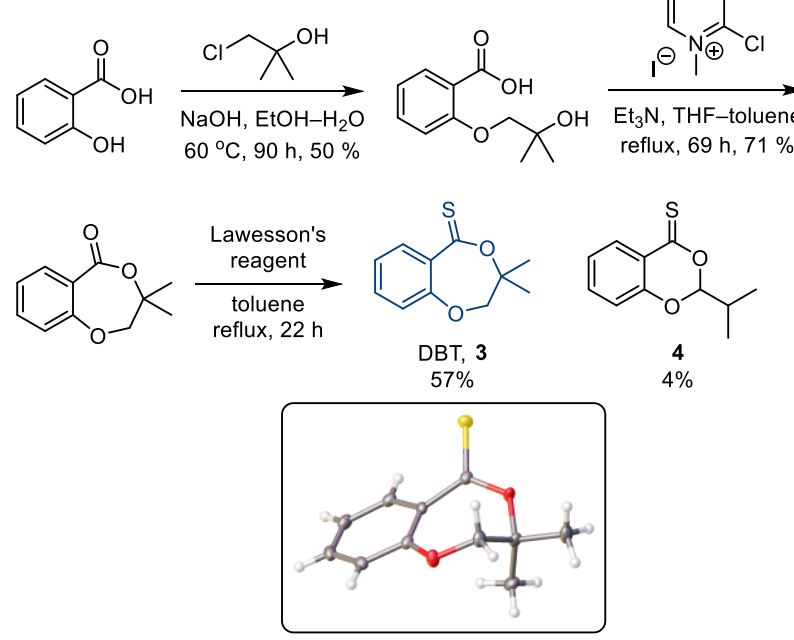

B-DBT Thiocarbonyl Addition-Ring-opening (TARO) Mechanism
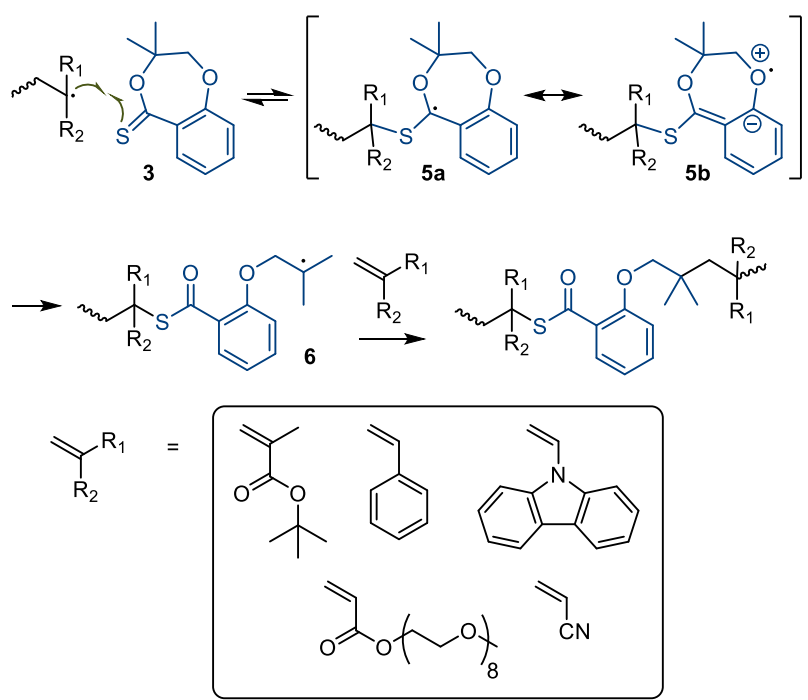

Scheme 1. (A) Synthesis of DBT, 3, with X-ray crystallographic structure $^{15}$ and (B) TARO copolymerization mechanism of DBT with resonance forms of the intermediate radical (5a-b) and structures of the compatible vinyl comonomers.

DBT was found to homopolymerize (Table S1, Figure S13S20) and copolymerize with acrylonitrile, poly(ethylene glycol) methyl ether acrylate, $N$-vinyl carbazole, styrene, and tert-butyl methacrylate (Figure S21-S35). The consumption of DBT was accompanied by the disappearance of its orange colour. Copolymers exhibited IR absorbances $\left(v_{\mathrm{C}-\mathrm{S}}\right.$ stretch $=899$ $\mathrm{cm}^{-1}, v_{\mathrm{C}=0}$ stretch $\left.=1671 \mathrm{~cm}^{-1}\right)^{16}$ and ${ }^{13} \mathrm{C}$ NMR resonances $(\delta \mathrm{s} C=0$ $=192 \mathrm{ppm}$ ) characteristic of thioesters, supporting the expected TARO mechanism and the formation of thioester backbone functionality. No evidence of ring-retaining polymerization was observed. Surprisingly, an attempted copolymerization of DBT with tert-butyl acrylate under RAFT conditions led to the homopolymerization of DBT (Figure S36-S37). This result is similar to the recently reported homopolymerization of $\varepsilon$-thionocaprolactone $\mathbf{2 a}$ in the presence of $50 \mathrm{~mol}-\% 2$ methylene-1,3-dioxepane. ${ }^{11}$ Unlike this literature precedent, however, DBT also homopolymerized in the absence of tertbutyl acrylate. An AIBN-initiated free-radical polymerization reached $35 \%$ conversion after heating to $70{ }^{\circ} \mathrm{C}$ overnight. Two subsequent additions of further AIBN followed by degassing increased the accumulated conversion to $66 \%$ and $88 \%$, respectively. Surprisingly, upon aminolysis, DBT homopolymer did not degrade into the expected salicylamide $O$-ether 7 , but $\mathrm{N}$-functional salicylamide and 2,2-dimethylthiirane, which were identified through ${ }^{1} \mathrm{H}$ and ${ }^{13} \mathrm{C}$ NMR measurements (Figure S38-S42). These results confirmed the presence of easily cleavable thioester backbone functionality and that the orthoalkoxy substituent provided an additional cleavable linkage (see Scheme 2).

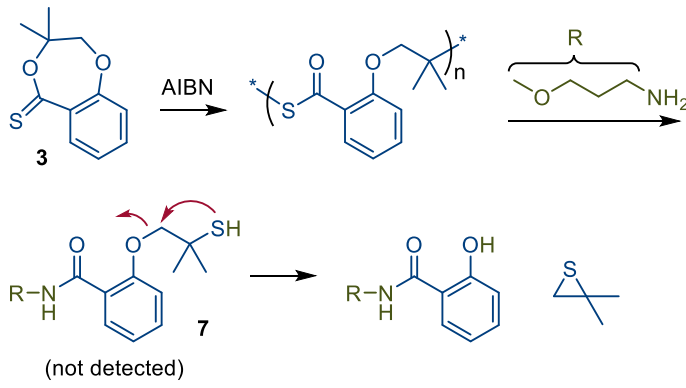

Scheme 2. Synthesis of DBT homopolymer and aminolysis with the expected (though not detected) primary degradation fragment (7) and proposed mechanism leading to the formation of the observed products.
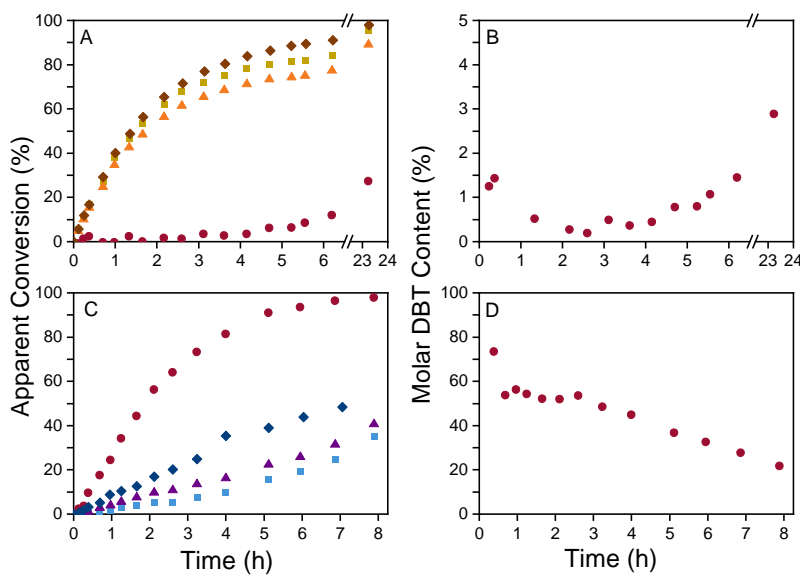

Figure 1. (A) Free-radical copolymerization kinetics of DBT$t$ BuMA (9:91 feed ratio) with the ${ }^{1} \mathrm{H}$ NMR-measured conversions of DBT (red circles) and $t$ BuMA (yellow squares), global conversion (orange triangles) and tBuMA conversion in a comparable homopolymerization (brown diamonds); (B) Estimated overall molar DBT content of the DBT- $t$ BuMA copolymer; (C) Free-radical copolymerization kinetics of DBTstyrene (9:91 feed ratio) with conversions of DBT (red circles) and styrene (light blue squares), global conversion (purple triangles) and styrene conversion in a comparable homopolymerization (dark blue diamonds); and (D) Estimated overall molar DBT content of the DBT-styrene copolymer.

Apart from homopolymerization, major advantages of DBT over DOT are its successful copolymerization with methacrylates and styrene. The AIBN-initiated free-radical copolymerization kinetics of DBT and tert-butyl methacrylate ( $t \mathrm{BuMA})$, as a representative methacrylic monomer, were determined for a 9:91 mol-\% DBT- $t$ BuMA feed ratio, see Figure 1A. The consumption of both comonomers was determined by ${ }^{1} \mathrm{H}$ NMR spectroscopy and followed first-order kinetics with estimated half-lives, $\mathrm{t}_{1 / 2}(t \mathrm{BuMA})=1.49 \mathrm{~h}$ and $\mathrm{t}_{1 / 2}(\mathrm{DBT})=49 \mathrm{~h}$. For comparison, a homopolymerization with a $100 \%$ tBuMA feed un- 
der the same conditions (and with the same overall monomer concentration $)$ had an observed $\mathrm{t}_{1 / 2}(t \mathrm{BuMAhomo})=1.43 \mathrm{~h}$ (Figure S43). This data indicated the uninhibited polymerization of $t$ BuMA in the presence of DBT with slow incorporation of the degradable repeat units. The DBT consumption increased as the $t$ BuMA feedstock was depleted and reached $27 \%$ after polymerizing overnight, corresponding to an overall DBT content of 3 mol-\%, see Figure 1B. This situation is a well-documented dilemma with cyclic ketene acetals (the most widely used RROP monomer type) showing similarly low reactivity not only with methacrylates but also acrylates and acrylamides. ${ }^{4}$ Higher incorporation can typically be forced through a higher feed of the cyclic monomer although this process still leads to a compositional gradient.

Next, a 23:77 mixture of DBT- $t$ BuMA was polymerized in the presence of 1 equiv of the trithiocarbonate RAFT agent 4cyano-4-[(dodecylsulfanylthiocarbonyl)sulfanyl]pentanoic acid yielding poly $\left(t \mathrm{BuMA}_{74}-\mathrm{co}-\mathrm{DBT}_{2}\right)$ with a measured $M_{\mathrm{n}} \mathrm{SEC}=$ $8.5 \mathrm{~kg} / \mathrm{mol}$ and $Ð=1.22$. This copolymer was then used as macro-RAFT agent for the chain extension with a $t$ BuMA homoblock (the same vinyl comonomer was chosen to facilitate SEC analysis) to give poly[( $\left.\left.t \mathrm{BuMA}_{74}-c o-\mathrm{DBT}_{2}\right)-b l-t \mathrm{BuMA}_{60}\right]$ $\left(M_{\mathrm{n}}{ }^{\mathrm{SEC}}=10.0 \mathrm{~kg} / \mathrm{mol}\right.$ and $\left.D=1.31\right)$. Upon treatment of the block copolymer with $N$-ethylamine in THF, a species with $M_{\mathrm{n}}{ }^{\mathrm{SEC}}=5.8 \mathrm{~kg} / \mathrm{mol}$ and $\emptyset=1.34$ was obtained, see Figure 2 . These results demonstrated that (i) TARO copolymerization of DBT was compatible with RAFT; the initial macro-RAFT agent had a low dispersity and retained the RAFT end group necessary for chain extension; (ii) all copolymers contained degradable backbone functionality with no residual block copolymer observed after aminolysis; (iii) the degradation product had a smaller apparent molar mass than the macro-RAFT agent, indicating that cleavage occurred within the first block rather than at the junction of the two blocks. RAFT polymerization has previously been demonstrated on thionolactones ${ }^{5}$ $8,10,11$ but the case of DBT warrants further consideration. Notably, the RAFT copolymerization was strongly retarded. The kinetics of a 9:91 mol-\% DBT- $t$ BuMA feed ratio gave estimated half-lives $\left(\mathrm{t}_{1 / 2}(t \mathrm{BuMA})=14 \mathrm{~h}\right.$ and $\left.\mathrm{t}_{1 / 2}(\mathrm{DBT})=159 \mathrm{~h}\right)$ much higher than a comparable homopolymerization of $t \mathrm{Bu}$ MA under RAFT conditions $\left(\mathrm{t}_{1 / 2}\left(t \mathrm{BuMA} \mathrm{A}_{\text {homo }}\right)=4.5 \mathrm{~h}\right)$ (Figure S44-S45). The retardation was presumed to arise from the attack of DBT-terminal chains on a RAFT agent because the DBT-derived radical (6) is a worse homolytic leaving group than the tertiary resonance-stabilised radical of a growing methacrylic chain. ${ }^{14}$ As the above results indicated, this did not lead to irreversible termination, but chains continued to grow (and could be extended) after adding the first DBT unit. It was hypothesised that the selective retardation of DBTterminal chains would lead to a more uniform distribution of thioester linkages between chains, because a DBT-terminal chain is likely to "wait" until most other chains have added a DBT unit, too, and symmetrical RAFT intermediates are formed. Even the low DBT content of poly( $t \mathrm{BuMA}_{\left.44-c o-\mathrm{DBT}_{2}\right)}$ (for which a Poisson distribution would result in $14 \%$ of chains with zero DBT units) could thus result in the observed degradability of all chains.
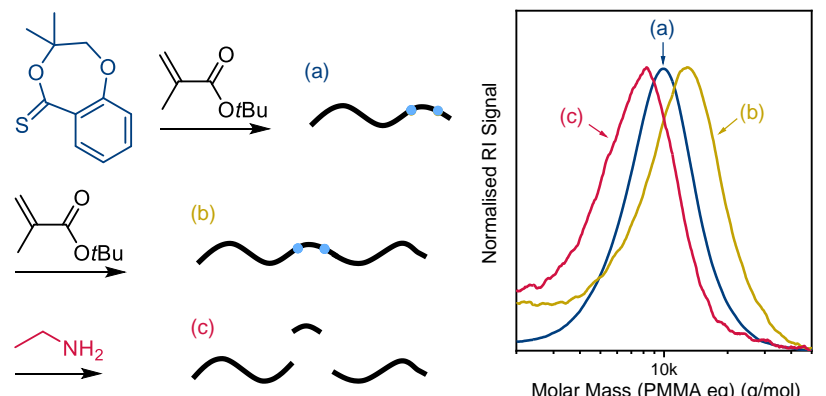

Figure 2. Size exclusion chromatograms of (a) RAFT-made poly $\left(t \mathrm{BuMA}_{74}-\mathrm{co}-\mathrm{DBT}_{2}\right)$, (b) poly[( $\left.t \mathrm{BuMA}_{74}-\mathrm{co}-\mathrm{DBT}_{2}\right)-b l-$ $t$ BuMA60], and (c) the products of the aminolysis of the diblock (right) with cartoon showing the expected positions of thioester groups (light blue circles) within the chains (left).

The copolymerization kinetics of 9:91 mol-\% DBT-styrene formulations, on the other hand, showed a strikingly different situation, see Figure 1C (free-radical polymerization) and Figure S47-S48 (RAFT). Compared to a comparable freeradical styrene (sty) homopolymerization $\left(t_{1 / 2}(\right.$ styhomo $\left.)=7.1 \mathrm{~h}\right)$, the presence of 9 mol-\% DBT in a freeradical copolymerization retarded the styrene uptake to $\mathrm{t}_{1 / 2}$ (sty) $=15 \mathrm{~h}$, while the DBT consumption was very rapid with a measured $t_{1 / 2}(D B T)=1.4 \mathrm{~h}$ (Figure S46). After $5 \mathrm{~h}$, the DBT conversion exceeded $90 \%$, while only $15 \%$ of styrene had been included into the oligomeric product. The estimated molar DBT content of the copolymer (Figure 1D) remained at $50 \%$ for the first $2.5 \mathrm{~h}$ of the copolymerization (suggesting the formation of alternating sequences) and became more styrene-rich as the DBT feed was being depleted. Similarly, a free radical copolymerization of a 10:90 mol-\% DBT-sty feed had $100 \%$ DBT and 38\% styrene conversions after running overnight and gave a product with an average 23 mol-\% DBT content and an SEC-measured bimodal size distribution, $M_{\mathrm{n}} \mathrm{SEC}=$ $8.2 \mathrm{~kg} / \mathrm{mol}$ and $Ð=2.53$. Upon aminolysis of the product, only part of the sample proved to be degradable with large chains remaining in the sample (see Figure $3 \mathrm{~A}$ ). This undegradable population was presumed to be polystyrene homopolymer formed after the DBT feed had been depleted. Conversely, adding the overall $10-\mathrm{mol}-\%$ DBT feed continuously into an active free-radical polymerization of styrene in a starve-fed (semi-batch) process ${ }^{17}$ resulted in a comparable styrene conversion of $42 \%$ but lower DBT conversion of $20 \%$. The product had an average DBT content of 5 mol-\%, and measured $M_{\mathrm{n}}{ }^{\mathrm{SEC}}=8.7 \mathrm{~kg} / \mathrm{mol}$ and $\emptyset=1.83$, showing a narrower size distribution than the batch sample. Gratifyingly, following aminolysis, SEC analysis showed a clear shift of the entire distribution and a lower $M_{\mathrm{n}}^{\mathrm{SEC}}=7.3 \mathrm{~kg} / \mathrm{mol}$ and $\oslash=1.82$, indicating that all chains had contained (an albeit small amount of) degradable DBT repeat units, see Figure 3B. While the process still requires optimisation to achieve a higher DBT content and more uniform distribution within chains (an elaborate process ${ }^{17}$ beyond the current scope), these results demonstrate the usefulness of a rapidly copolymerizing thionolactone to produce degradable styrene copolymers through freeradical polymerization and without the need of RAFT agents. 


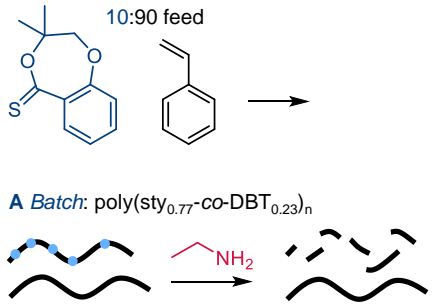

B Semi-batch: poly $\left(\text { sty }_{0.95}{ }^{-c o}-\text { DBT }_{0.05}\right)_{\mathrm{m}}$ (continuous addition of DBT)
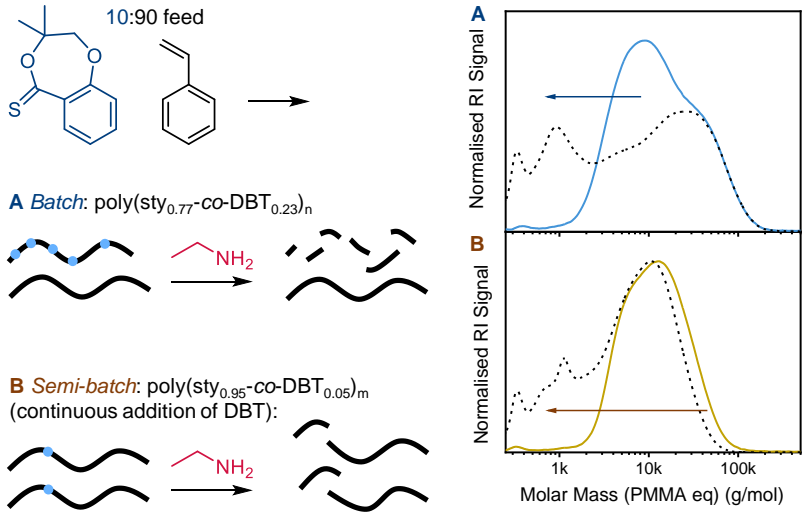

Figure 3. Size exclusion chromatograms (right, where dashed curves show measurements done on aminolysed samples) and cartoon (left) for the free-radical copolymerization of DBT and styrene in batch (all monomers together, A) and in semi-batch (B) where DBT was added continuously during the polymerization.

Concluding, structural modifications of the previously described thionolactone DOT resulted in drastically different (co)polymerization properties. Specifically, DBT outperformed DOT in three key challenges. Firstly, DBT formed homopolymers that showed an unexpected self-degradation of the ether bond. Secondly, DBT copolymerized with methacrylates. Although the incorporation of thioesters was sluggish and RAFT polymerization significantly retarded, the high energy re-initiating radical of DBT was hypothesised to cause to selective retardation that led to a more uniform distribution of degradable thioesters between chains. Thirdly, the very rapid incorporation of DBT into styrene copolymers was exploited through a starve-fed setup to produce degradable copolymers through free-radical polymerization. These novelties demonstrate that cyclic thionocarbonyl monomers can be adapted to be compatible with other monomer types, broadening the scope and applicability of TARO radical polymerization.

\section{ASSOCIATED CONTENT}

\section{Supporting Information}

Experimental section, homo- and copolymer NMR and IR data, copolymerization kinetics, homo- and copolymer degradation data.

CCDC 2124098 contains the supplementary crystallographic data for this paper. The data can be obtained free of charge via www.ccdc.cam.ac.uk/data_request/cif, or by emailing data_request@ccdc.cam.ac.uk, or by contacting The Cambridge Crystallographic Data Centre, 12 Union Road, Cambridge CB2 1EZ, UK; fax: +44 1223336033

\section{AUTHOR INFORMATION}

\section{Corresponding Author}

email address p.roth@surrey.ac.uk

\section{Notes}

The authors declare no competing financial interests.

\section{ACKNOWLEDGMENT}

The authors gratefully acknowledge studentship funding for M. F. I. Rix from the University of Surrey and from Syngenta, and Dr Annette Christie, Dr Nick Mulholland, and Dr Chris Lindsay (Syngenta) for continued support. Kyle Collins (University of Surrey) is acknowledged for assistance with computational calculations.

\section{REFERENCES}

1. Delplace, V.; Nicolas, J., Degradable vinyl polymers for biomedical applications. Nature Chemistry 2015, 7 (10), 771.

2. Pesenti, T.; Nicolas, J., 100th Anniversary of Macromolecular Science Viewpoint: Degradable Polymers from Radical RingOpening Polymerization: Latest Advances, New Directions, and Ongoing Challenges. ACS Macro Letters 2020, 9 (12), 1812-1835.

3. Jackson, A. W., Reversible-deactivation radical polymerization of cyclic ketene acetals. Polym. Chem. 2020, 11 (21), 3525-3545.

4. Tardy; Nicolas; Gigmes; Lefay; Guillaneuf, Radical Ring-Opening Polymerization: Scope, Limitations, and Application to (Bio)Degradable Materials. Chem Rev 2017, 117 (3), 1319.

5. Bingham, N. M.; Roth, P. J., Degradable vinyl copolymers through thiocarbonyl addition-ring-opening (TARO) polymerization. Chem Commun (Camb) 2019, 55 (1), 55-58.

6. Barton, D. H. R.; Crich, D.; Löbberding, A.; Zard, S. Z., On the mechanism of the deoxygenation of secondary alcohols by the reduction of their methyl xanthates by tin hydrides. Tetrahedron 1986, 42 (8), 2329-2338.

7. Bingham, N. M.; Nisa, Q. u.; Chua, S. H. L.; Fontugne, L.; Spick, M. P.; Roth, P. J., Thioester-Functional Polyacrylamides: Rapid Selective Backbone Degradation Triggers Solubility Switch Based on Aqueous Lower Critical Solution Temperature/Upper Critical Solution Temperature. ACS Applied Polymer Materials 2020, 2 (8), 3440-3449.

8. Smith, R. A.; Fu, G.; McAteer, O.; Xu, M.; Gutekunst, W. R., Radical Approach to Thioester-Containing Polymers. Journal of the American Chemical Society 2019, 141 (4), 1446-1451.

9. Spick, M. P.; Bingham, N. M.; Li, Y.; de Jesus, J.; Costa, C.; Bailey, M. J.; Roth, P. J., Fully Degradable Thioester-Functional Homoand Alternating Copolymers Prepared through Thiocarbonyl Addition-Ring-Opening RAFT Radical Polymerization. Macromolecules 2020, 53 (2), 539-547.

10. Ivanchenko, O.; Authesserre, U.; Coste, G.; Mazières, S.; Destarac, M.; Harrisson, S., $\varepsilon$-Thionocaprolactone: an accessible monomer for preparation of degradable poly(vinyl esters) by radical ring-opening polymerization. Polymer Chemistry 2021, 12 (13), 1931-1938.

11. Plummer, C. M.; Gil, N.; Dufils, P.-E.; Wilson, D. J.; Lefay, C.; Gigmes, D.; Guillaneuf, Y., Mechanistic Investigation of $\varepsilon$-ThionoCaprolactone Radical Polymerization: An Interesting Tool to Insert Weak Bonds into Poly(vinyl esters). ACS Applied Polymer Materials 2021, 3 (6), 3264-3271.

12. Moad, G., Mechanism and Kinetics of Dithiobenzoate-Mediated RAFT Polymerization - Status of the Dilemma. Macromolecular Chemistry and Physics 2014, 215 (1), 9-26.

13. Moad, G., Mechanism and Kinetics of Dithiobenzoate-Mediated RAFT Polymerization - Status of the Dilemma. Macromolecular Chemistry and Physics 2017, 218 (19).

14. Hioe, J.; Zipse, H., Radical Stability-Thermochemical Aspects. In Encyclopedia of Radicals in Chemistry, Biology and Materials, 2012.

15. Coles, S. J.; Gale, P. A., Changing and challenging times for service crystallography. Chemical Science 2012, 3 (3), 683-689.

16. Crowder, G. A., The C-S Stretching Frequency in Thiol Acids and Esters. Applied Spectroscopy 1972, 26 (4), 486-487.

17. Lena, J.-B.; Jackson, A. W.; Chennamaneni, L. R.; Wong, C. T.; Lim, F.; Andriani, Y.; Thoniyot, P.; Van Herk, A. M., Degradable Poly(alkyl acrylates) with Uniform Insertion of Ester Bonds, Comparing Batch and Semibatch Copolymerizations. Macromolecules 2020, 53 (10), 3994-4011. 
A novel thionolactone is shown to undergo free and RAFT radical homopolymerization and copolymerization with styrene and methacrylate comonomers, providing copolymers with selectively degradable thioester backbone functionality.

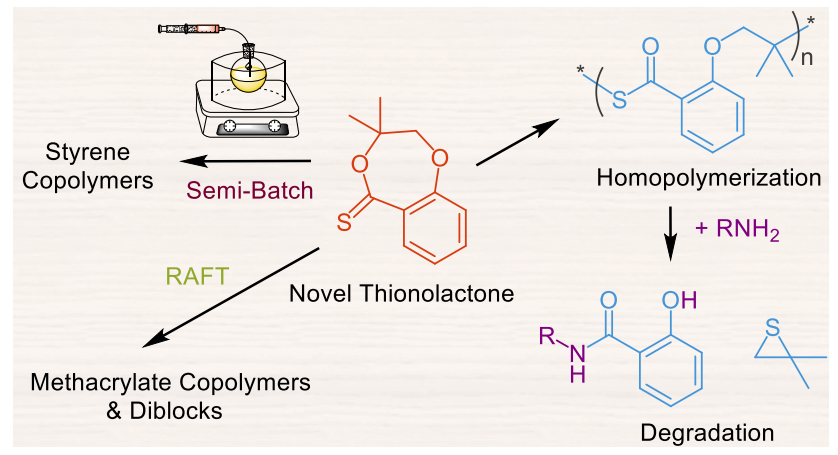

